\title{
Postprint
}

This document is the Accepted Manuscript version of a Published Work that appeared in final form in Nano Letters

after peer review and technical editing by the publisher.

To access the final edited and published work see:

Sihan Zhao, Gabriela Borin Barin, Ting Cao, Jan Overbeck, Rimah Darawish, Tairu Lyu, Steve Drapcho, Sheng Wang, Tim Dumslaff, Akimitsu Narita, Michel Calame, Klaus Müllen, Steven G. Louie, Pascal Ruffieux, Roman Fasel and Feng Wang

Optical imaging and spectroscopy of atomically precise armchair graphene nanoribbons

Nano Lett. 2020, 20, 2, 1124-1130

https://pubs.acs.org/doi/10.1021/acs.nanolett.9b04497

Access to the published version may require subscription.

When citing this work, please cite the original published paper. 


\section{Optical imaging and spectroscopy of atomically precise armchair graphene nanoribbons}

Sihan Zhao ${ }^{1}$, Gabriela Borin Barin ${ }^{2}$, Ting Cao ${ }^{1,3}$, Jan Overbeck ${ }^{2}$, Rimah Darawish ${ }^{2}$, Tairu Lyu ${ }^{1}$, Steve Drapcho $^{1}$, Sheng Wang ${ }^{1,4}$, Tim Dumslaff ${ }^{5}$, Akimitsu Narita ${ }^{5}$, Michel Calame ${ }^{2}$, Klaus Müllen ${ }^{5,6}$, Steven G. Louie $^{1,4}$, Pascal Ruffieux ${ }^{2}$, Roman Fasel ${ }^{2,7}$ and Feng Wang ${ }^{1,4,8^{*}}$

1. Department of Physics, University of California at Berkeley, Berkeley, California 94720, United States

2. Empa, Swiss Federal Laboratories for Materials Science and Technology, 8600 Dübendorf, Switzerland

3. Department of Materials Science and Engineering, University of Washington, Seattle, WA, USA

4. Materials Science Division, Lawrence Berkeley National Laboratory, Berkeley, California 94720, United States

5. Max Planck Institute for Polymer Research, Ackermannweg 10, D-55128 Mainz, Germany

6. Johannes Gutenberg-Universität Mainz, Institute of Physical Chemistry, 5128 Mainz, Germany

7. Department of Chemistry and Biochemistry, University of Bern, Freiestrasse 3, CH-3012 Bern, Switzerland

8. Kavli Energy NanoSciences Institute at the University of California, Berkeley and the Lawrence Berkeley National Laboratory

Corresponding author: fengwang76@berkeley.edu

Abstract: We report the optical imaging and absorption spectroscopy on atomically precise armchair graphene nanoribbons (GNRs) on insulating fused silica substrates. This is achieved by controlling light polarization on macroscopically aligned GNRs, which greatly enhances the sub-monolayer GNR optical contrast on the insulating substrates. We measured the linear absorption spectra of 7-armchair and 9armchair GNRs in this study, and the experimental data agree qualitatively with ab inito calculation results. The polarization spectroscopy technique enables an unambiguous optical identification of GNR samples and provides a rapid tool to characterize the transferred film over large area.

Key words: graphene nanoribbons, armchair graphene nanoribbons, optical imaging, optical properties, absorption spectroscopy, polarization spectroscopy

Recent advances in bottom-up synthesis of graphene nanoribbons (GNRs) have led to realization of atomically precise GNRs with well-defined edge structures. ${ }^{1-16}$ The unprecedented structural control with atomic precision opens new opportunities to engineer GNR bandstructures, making GNRs a promising material platform for nanodevices. ${ }^{15-20}$ Armchair GNRs (AGNRs) are the most extensively studied atomically precise GNRs. AGNRs with different widths (N-AGNRs, where $\mathrm{N}$ represents the number of carbon dimer lines across the GNR width) were synthesized and they are structurally stable in ambient conditions. ${ }^{1,5,10,18,19,21,22}$ Theoretically, the one-particle bandgap in AGNRs varies with the nanoribbon width following distinct family patterns: the bandgap in N-AGNRs does not decrease monotonically with the GNR width, but oscillates periodically with the family index $\mathrm{i}=\mathrm{N} \bmod 3 .^{23-26}$

Most of the sample characterizations so far are carried out by scanning tunneling microscopy (STM) in situ on gold and Raman spectroscopy ex situ. ${ }^{1,5,10,27-29}$ However, the linear optical absorption which characterizes the fundamental light-matter interactions of transferred AGNRs on insulating substrates have remained challenging. ${ }^{22,30-33}$ Limited absorption studies were previously reported for monolayer AGNRs on gold surfaces and for stacked multilayer AGNRs on insulating substrates, which, however, involved complicated metal screening effect and coupling effect among AGNR stacks. ${ }^{22,30-33}$ Absorption 
spectroscopy study of the intrinsic one-dimensional (1D) AGNRs is hindered by a combination of impurities/residues introduced in the transfer process (e.g. fig. 1c) and the intrinsic weak optical absorption of the sub-monolayer 1D objects.

Here we report the first optical imaging and absorption spectroscopy for sub-monolayer atomically precise AGNRs with different widths on insulating substrates. These are achieved by applying a light polarization spectroscopy technique on macroscopically aligned AGNR samples, where the weak optical contrast of GNRs is greatly enhanced with the suppressed background signal from substrate and from impurities/residues. We experimentally obtain absorption spectra from 7-AGNRs and 9-AGNRs transferred on fused silica substrates whose results qualitatively agree with our theoretical GW-BSE calculations. ${ }^{1,13}$ We experimentally observe optical bandgaps of $\sim 2.3 \mathrm{eV}$ for 7-AGNRs and $\sim 1.3 \mathrm{eV}$ for 9-AGNRs.

\section{Results and discussion}

a

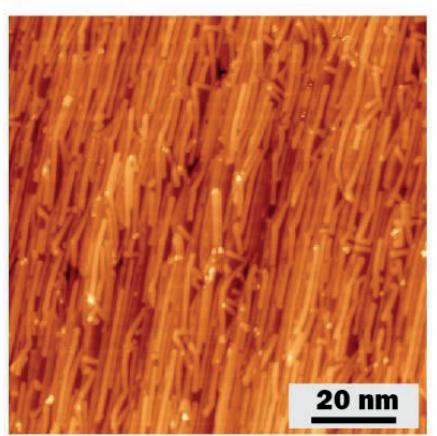

C

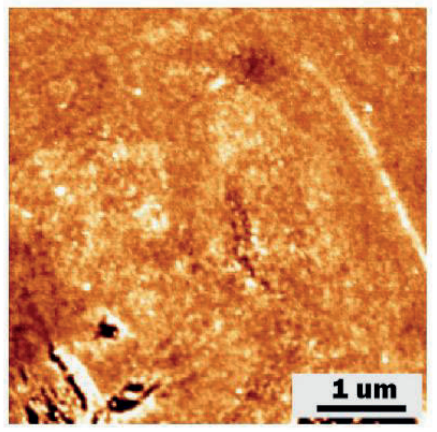

b

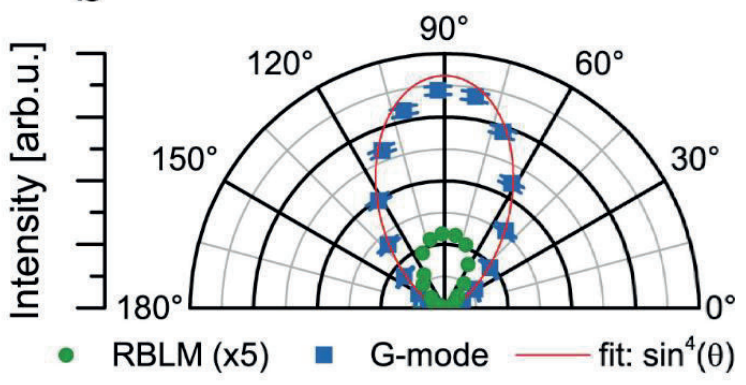

d

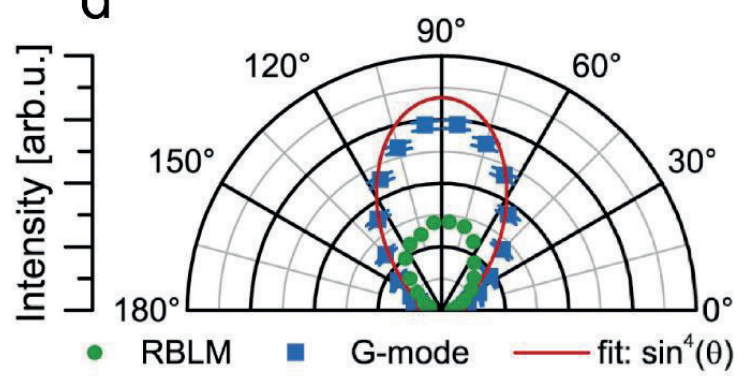

Figure 1. Microscopy and spectroscopy characterizations of aligned 7-AGNRs before and after transfer. (a) STM topology image for the as-grown aligned 7-AGNRs on Au $(11,12,12)$. (b) Polarization-resolved Raman of G- and RBLM-modes for as-grown aligned 7-AGNRs. The data (e.g. G-mode) nicely follow a $\sin ^{4} \theta$ fit, with an extracted polarization anisotropy of 0.90 . (c) AFM topology image for the transferred aligned 7-AGNRs on insulating substrate. It shows GNR film non-uniformity and residues/impurities incurred during the transfer process. (d) Polarizationresolved Raman of G- and RBLM-modes for the transferred aligned 7-AGNRs. The polarization anisotropy after transfer is 0.82 indicating that the macroscopic alignment of GNRs is largely preserved after transfer process. $532 \mathrm{~nm}$ laser excitation is used in (b) and (d).

The aligned AGNRs samples are directly grown on $\mathrm{Au}(11,12,12)$ and/or $\mathrm{Au}(7,8,8)$ (Methods section 1). Figure 1a shows a representative STM topology image of the as-grown 7-AGNRs, where the individual 
GNRs exhibit a unidirectional alignment direction on along the $\mathrm{Au}(11,12,12)$ terraces. The width of each 7-AGNRs is $\sim 1 \mathrm{~nm}$ and their average length is $\sim 30 \mathrm{~nm}$. Figure $1 \mathrm{~b}$ shows the polarization-resolved Raman intensity of the G-mode $\left(\sim 1600 \mathrm{~cm}^{-1}\right)$ and RBLM-mode $\left(\sim 400 \mathrm{~cm}^{-1}\right)$ for the as-grown 7-AGNRs. The data nicely follow $\sin ^{4} \theta$, where $\theta$ denotes the relative angle between light polarization and AGNR aligned axis and thus confirm that the as-grown 7-AGNRs are macroscopically aligned ${ }^{28}$ To probe the intrinsic optical properties, we transfer AGNRs onto transparent and insulating substrate (i.e. fused silica which is amorphous) by using an electrochemical delamination process (also known as "bubble transfer") (Methods section 2). ${ }^{28}$ We characterize the morphology of the as-transferred GNRs by atomic force microscopy (AFM). Figure 1c shows the typical GNR film after transfer. We note that there exists GNR film nonuniformity and residues/impurities induced in the transfer process (fig. 1c). Figure 1d presents the polarization-resolved Raman intensity of G- and RBLM-modes for the transferred 7-AGNRs. For a quantitative description of the alignment, we calculate the Raman polarization anisotropy $P$ for the G-mode as $P=\left(I_{\|}-I_{\perp}\right) /\left(I_{\|}+I_{\perp}\right)$. This yields $P=1$ for GNRs that are perfectly aligned with the step edges and $P=0$ for randomly oriented GNRs. ${ }^{28}$ For the as-grown 7-AGNRs on $\mathrm{Au}(11,12,12)$ we find an anisotropy of $\mathrm{P}=$ 0.90 (fig. 1b), which slightly decreases to $\mathrm{P}=0.82$ (fig. 1d) after transfer, indicating that the macroscopic alignment is largely preserved upon transfer.

A combination of the weak optical absorption of the sub-monolayer GNRs and the background from unavoidable impurities/residues introduced in transfer process makes the absorption spectroscopy of transferred GNRs on an insulating substrate very challenging. To overcome this difficulty, we exploit a polarization spectroscopy technique on these 1D aligned AGNRs. Figures 2a and $2 b$ illustrate our optical setup and measurement scheme of the polarization spectroscopy technique. From an interferometric point of view, the optical contrast of GNRs on a substrate in a reflection configuration results from interference between the GNR-reflected electric field $E_{G N R}$ and the substrate-reflected electric field $E_{s u b}$ at the detector site:

$$
d R / R=\frac{\left|E_{G N R}+E_{\text {sub }}\right|^{2}-\left|E_{\text {sub }}\right|^{2}}{\left|E_{\text {sub }}\right|^{2}} \approx \frac{2 R e\left[E_{\text {sub }}^{*} E_{G N R}\right]}{\left|E_{\text {sub }}\right|^{2}}=\frac{2\left|E_{G N R}\right|}{\left|E_{\text {sub }}\right|} \cos \theta
$$

Here $R$ is the reflected optical signal from the substrate, $d R$ is the reflected optical signal difference with the presence of GNRs, and $\theta$ represents the relative phase difference between $E_{G N R}$ and $E_{S u b}$. We treat $E_{G N R}$ as a perturbation of $E_{\text {sub }}$ because it is usually much smaller comparing with $E_{\text {sub }}$. Therefore, in principle, the GNR optical contrast can be significantly enhanced by reducing the reflected electric field from substrate while maintaining the electric field from GNRs. This is achieved by polarization manipulation, as shown in fig. $2 \mathrm{a}$ and $2 \mathrm{~b}$. The horizontally polarized incident light after the first polarizer P1 illuminates the macroscopically-aligned GNR samples on fused silica substrate. To maximize the GNR optical contrast, the incident laser polarization is at about $45^{\circ}$ relative to the GNR axis (fig. 2b). The GNRreflected electric field at sample site $E_{G N R}^{0}$ is mostly polarized along the GNR axis direction due to the strong depolarization effect in the direction perpendicular to GNR axis. ${ }^{34,35}$ On the other hand, the electric field reflected from the isotropic insulating substrate at the substrate site $E_{\text {sub }}^{0}$ remains the horizontal polarization direction. The second polarizer P2 is set nearly orthogonal to P1 (with a small deviation angle $\delta$ shown in fig. $2 \mathrm{~b}$ ) in the outgoing beam path. The substrate-reflected field (as well as those from impurities/residues incurred during sample transfer) is thus significantly reduced by $\sin \delta * E_{s u b}^{0}$. In contrast, the GNR-reflected electric field after P2 is still largely kept (reduced by $1 / \sqrt{2} E_{G N R}^{0}$ ). Therefore, the GNR optical contrast is greatly enhanced by $1 /(\sqrt{2} \sin \delta)$ against signals from substrate and impurities/residues.

The substrate used in this study is insulating and transparent fused silica substrate with semi-infinite thickness $(\sim 500 \mu \mathrm{m})$. In this case, the measured GNR optical contrast in eq 1 is proportional to $\operatorname{Im}(\chi) / \sin \delta$ when $\delta$ is small, where $\chi$ is the optical susceptibility of GNRs. This yields a direct 
measurement of GNR optical absorption. The sign of optical contrast depends on the angle $\delta$. The GNRs can appear brighter or darker than the environment (i.e. substrate) depending on the sign of the deviation angle from vertical direction (fig. $2 \mathrm{~b}$ illustrates a small positive $\delta$ ). By gradually decreasing the magnitude of $\delta$, GNR optical contrast will show a monotonic increase (Methods section 3). ${ }^{36}$
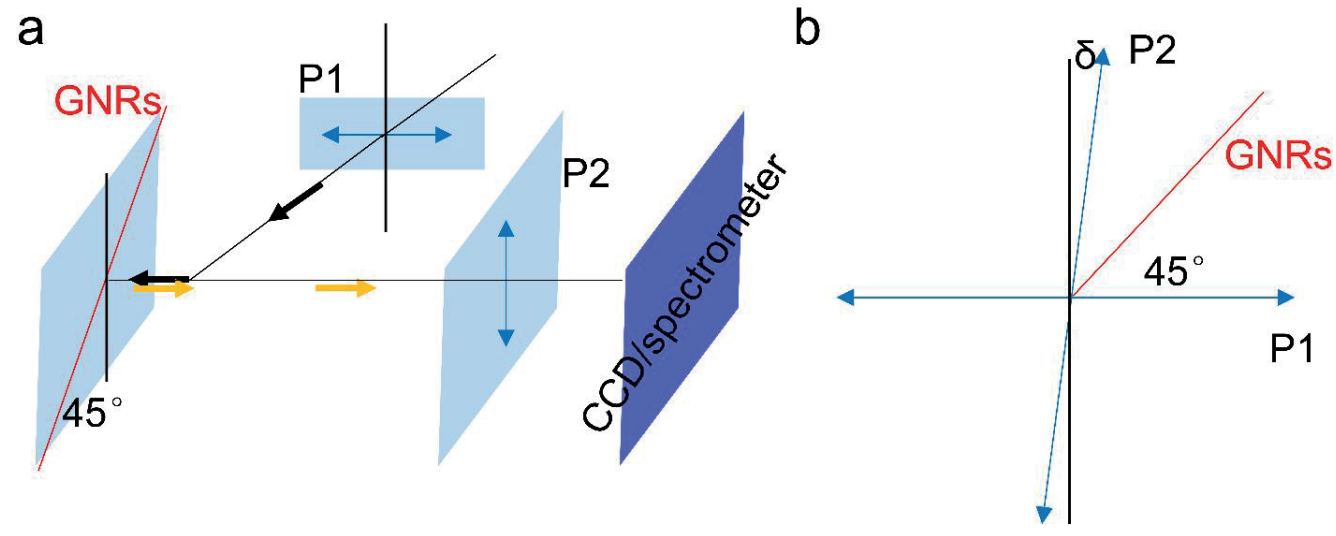

C

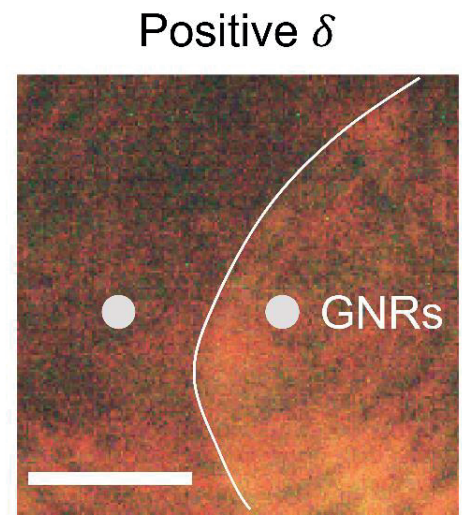

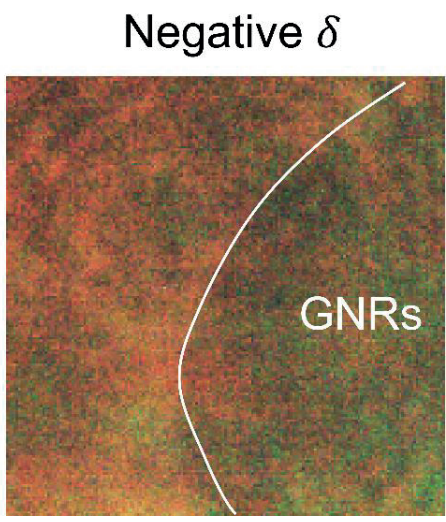

Figure 2. Application of the polarization spectroscopy technique in aligned AGNRs. (a-b) Schematic illustration of our optical setup and measurement scheme. To maximize the GNR optical contrast, GNR axis is at about $45^{\circ}$ to the first polarizer P1. The second polarizer P2 is nearly orthogonal to $\mathrm{P} 1$ with a deviation angle defined as $\delta$. The optical signals from substrates and impurities/residues are significantly reduced when $\delta$ is set small, whereas, on the other hand, signals from GNRs are largely kept. (c) Optical imaging of transferred GNR samples on fused silica substrate with the polarization spectroscopy technique as schematically illustrated in (a) and (b). The left and right are two microscope images corresponding to imaging conditions with $\delta$ at $\sim 2^{\circ}$ and at $\sim-2^{\circ}$, respectively. The dashed lines are boundary contours separating the bare substrate on the left and GNRs on the right. GNRs appear either brighter (left image) or darker (right image) comparing with the substrate when $\delta$ is changed from positive values to negative ones. The measuring spots where the spectroscopy data are obtained in fig. $3 \mathrm{a}$ are shown by dot markers. Scale bar is about $10 \mu \mathrm{m}$. The ring-like fringes in the images are imaging artifacts due to the small opening of the iris used to block the stray light.

Our polarization spectroscopy technique enables a direct optical imaging of GNRs on fused silica substrate. Figure $2 \mathrm{c}$ shows the typical imaging results for the aligned 7-AGNR samples that were transferred. The image on the left is obtained when $\delta$ is set at $\sim 2^{\circ}$ whereas the one on the right is obtained when $\delta$ is set at $\sim-2^{\circ}$. The ring-like fringes in two images are imaging artifacts due to the small opening of the iris used to block the stray light. We clearly see that the optical contrast in two images changes sign when $\delta$ 
crosses zero: the brighter (darker) region becomes darker (brighter) when $\delta$ is changed from $\sim 2^{\circ}$ to $\sim-2^{\circ}$. In addition, the optical contrast becomes higher with decreasing the magnitude of $\delta$ for both positive and negative values. These unique behaviors are assigned to the GNRs' optical response arising from its unique 1D nature. The contours separating two distinct contrast regions are indicated by dashed lines. The GNR optical contrast on substrate is significantly enhanced with this polarization manipulation, and can achieve to more than $10 \%$ depending on the local densities of transferred GNR films. We detected GNR Raman signals over the brighter (darker) contrast region on the left (right) images and that no GNR Raman features were observed in the corresponding darker (brighter) contrast region (Figure S1), which further confirms the successful optical imaging and identification of GNRs on substrates with the polarization spectroscopy technique. Note that we always observe contrast variations within the transferred film area by this polarization spectroscopy technique, whereas, on the other hand, the film usually appears optically invisible and featureless under conventional optical microscope. The enhanced GNR optical contrast against the substrate thus enables an unambiguous optical identification of GNR samples and provides a rapid tool to characterize the transferred film over large area.

In the following, we will focus on the reflection contrast spectroscopy of aligned 7-AGNRs and 9AGNRs, which are representative AGNRs belonging to $3 p+1$ and $3 p$ family respectively. The optical spectra in this study are taken from $\sim 0.7 \mathrm{eV}$ to $\sim 2.8 \mathrm{eV}$, limited by the spectral range of our supercontinuum laser. Figure 3a representatively presents reflection contrast spectra $(d R / R)$ for 7-AGNRs. The measuring spots for $d R / R$ in fig. $3 \mathrm{a}$ are indicated by the dot markers in fig. 2c. Spectra measured with different deviation angles $\delta$ are plotted with different colors. Consistent spectra are observed at different sample locations (Figure S2). As shown in fig. 3a, a systematic evolution of $d R / R$ spectra with varying the deviation angles is observed: i) the measured $d R / R$ is positive (negative) when $\delta$ is positive (negative) and they exhibit a sign change when $\delta$ crosses zero; ii) by gradually decreasing the magnitude of deviation angles, we observe a monotonic increase in the magnitude of $d R / R$. These systematic behaviors unambiguously demonstrate the measured $d R / R$ arise from the GNR optical contrast and correspond to its optical absorption.
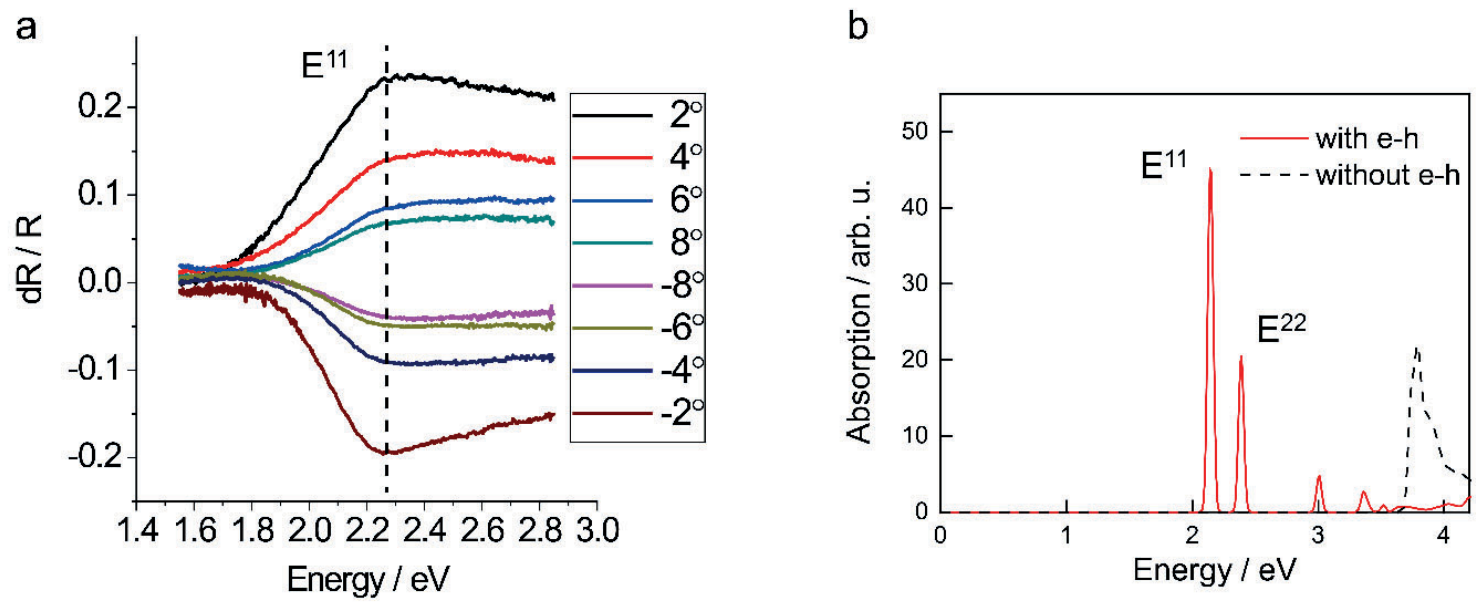

Figure 3. Experimental results and theoretical calculations on the optical absorption of 7-AGNRs. (a) Reflection contrast spectra $(d R / R)$ as a function of deviation angle $\delta$. $\mathrm{E}^{11}$ transition arising from the first bright exciton is indicated by the dashed line. (b) Theoretically calculated absorption spectra for 7-AGNRs with e-h interactions (solid red) and without e-h interactions (dashed black). The experimentally observed optical bandgap for 7AGNRs on fused silica substrate is $\sim 2.3 \mathrm{eV}$.

We focus on $d R / R$ spectra at deviation angles of $\sim 2^{\circ}$ and $\sim-2^{\circ}$ because the GNR optical contrasts at these angles are mostly enhanced against signals from substrates and from residues/impurities. The strong 
optical resonance observed at $\sim 2.3 \mathrm{eV}$ (dashed line) is attributed to the bright exciton from first intersubband $\left(\mathrm{E}^{11}\right)$ transition in 7-AGNRs. The strongly asymmetric spectral shape of $\mathrm{E}^{11}$ is likely due to higher energy bright exciton transition $\left(E^{22}\right)$ in 7-AGNRs. However, the very broad optical transition in the experimental spectrum prevents us to fully resolve multiple exciton resonances that may be present. The experimental interpretation on the observed spectra is supported by our theoretical GW-BSE calculation. Figure $3 \mathrm{~b}$ shows the calculated absorption spectra for 7-AGNRs with electron-hole (e-h) interactions (solid red) and without e-h interactions (dashed black). The calculated GW bandstructure for 7-AGNR is shown in Figure S3. Theoretically, the strong e-h coulomb interactions largely modify the optical absorption spectrum of 7-AGNRs, which causes a significant energy redshift for $\mathrm{E}^{11}$ from $\sim 3.7 \mathrm{eV}$ to $\sim 2.1 \mathrm{eV}$ as shown in fig. $3 b .{ }^{37,38}$ Our calculation predicts that 7-AGNRs have two bright exciton transitions $\mathrm{E}^{11}$ and $\mathrm{E}^{22}$ that are close in energy. Our experimentally observed absorption spectra and interpretation (fig. 3a) thus shows a qualitative agreement with the GW-BSE calculated spectra. The optical bandgap (i.e. the energy of $\mathrm{E}^{11}$ ) for 7-AGNRs on fused silica is experimentally determined to be $\sim 2.3 \mathrm{eV}$.

a

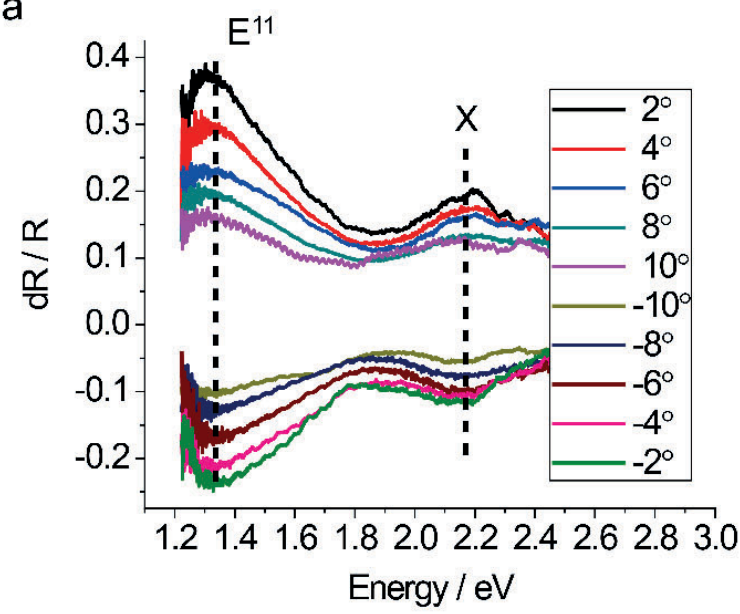

b

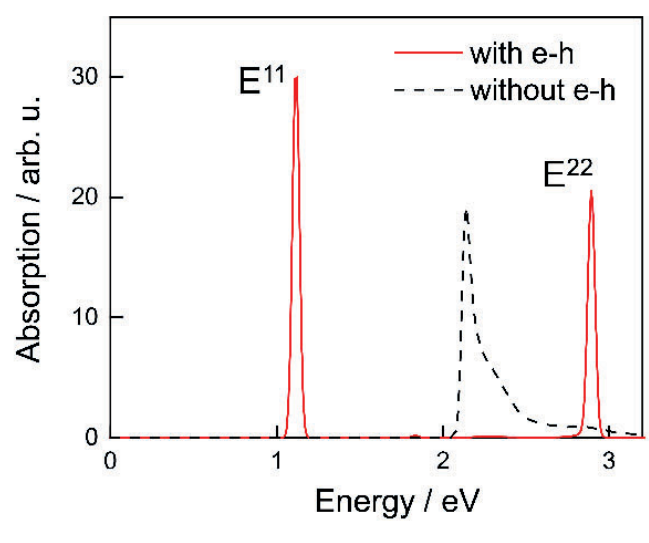

Figure 4. Experimental results and theoretical calculations on the optical absorption of 9-AGNRs. (a) Reflection contrast spectra $(d R / R)$ as a function of deviation angle $\delta$. The transition associated with the first bright exciton $\left(\mathrm{E}^{11}\right)$ and an $\mathrm{X}$ peak are indicated by dashed lines. (b) Theoretically calculated absorption spectra for 9-AGNRs with e-h interactions (solid red) and without e-h interactions (dashed). The experimentally observed optical bandgap for 9-AGNRs on fused silica substrate is $\sim 1.3 \mathrm{eV}$.

9-AGNRs are predicted to have a smaller bandgap compared to the 7-AGNRs. The representative $d R / R$ spectra for aligned 9-AGNRs with systematically varying deviation angles are presented in fig. 4a (more absorption data in Figure S4). The calculated absorption spectra with and without e-h interactions for 9-AGNRs are shown in fig. 4b. The calculated GW bandstructure is shown in Figure S5. Similar to the spectra of 7-AGNRs in fig. 3a, a systematic evolution of $d R / R$ on deviation angles is observed for 9AGNRs over the whole spectral range. We observe a very strong optical resonance at $\sim 1.3 \mathrm{eV}$. Consistent optical absorption spectra around $\sim 1.3 \mathrm{eV}$ resonant peak are confirmed with extending spectral range to lower energy by using an InGaAs detector (Figure S6). We attribute this strong optical resonance at $\sim 1.3$ $\mathrm{eV}$ to the bright $\mathrm{E}^{11}$ transition in 9-AGNRs. This assignment agrees with the GW-BSE calculated $\mathrm{E}^{11}$ transition with taking into account of e-h interactions (solid red in fig. 4b). The optical bandgap for 9AGNRs on fused silica is thus experimentally determined to be $\sim 1.3 \mathrm{eV}$. We also observe a weaker absorption peak (X peak in fig. 4a) at $\sim 2.2 \mathrm{eV}$ for 9-AGNR samples. This absorption peak locates at a much lower energy than the theoretically predicted $\mathrm{E}^{22}$ bright exciton transition (see fig. $4 \mathrm{~b}$ ). We do not 
know the origin of this absorption peak yet, and further theoretical and experimental studies will be required for its understanding.

In summary, by using the polarization spectroscopy technique, we successfully performed the optical imaging and absorption spectroscopy on two representative atomically precise AGNRs (i.e. 7-AGNRs and 9-AGNRs) with sub-monolayer thickness on insulating substrates. The unambiguous optical identification of GNRs and the systematic spectral evolution upon varying the polarization conditions consolidate that our observed spectroscopy results correspond to the 1D GNRs absorption. The experimentally observed absorption spectra and interpretation qualitatively agree with the GW-BSE calculations. The experimentally observed optical bandgaps for 7-AGNRs and 9-AGNRs on fused silica substrate are at $\sim 2.3 \mathrm{eV}$ and at $\sim$ $1.3 \mathrm{eV}$.

\section{Methods}

\section{Growth and STM characterization of aligned AGNRs}

\section{On surface synthesis of AGNRs}

9-AGNRs were synthesized from 3',6'-diiodo-1,1':2',1"-terphenyl (DITP) as the precursor monomer and 7AGNR from 10,10' -dibromo-9,9' -bianthryl (DBBA). ${ }^{39}$ In this study, 7-AGNRs and 9-AGNRs were grown on $\mathrm{Au}(11,12,12)$ and $\mathrm{Au}(788)$, respectively. First, $\mathrm{Au}(11,12,12)$ or $\mathrm{Au}(788)$ substrate was cleaned in ultra-high vacuum by two sputtering/annealing cycles: $1 \mathrm{kV} \mathrm{Ar}{ }^{+}$for 10 minutes followed by annealing at $420{ }^{\circ} \mathrm{C}$ for 10 minutes. Next, the monomer was sublimed onto the Au surface from a quartz crucible heated to $70{ }^{\circ} \mathrm{C}$ (DITP) and $200{ }^{\circ} \mathrm{C}$ (DBBA) respectively, with the substrate held at room temperature. After 1 monolayer coverage deposition, for both 7-, and 9-AGNRs the substrate was heated $\left(0.5^{\circ} \mathrm{C} / \mathrm{s}\right)$ up to $200{ }^{\circ} \mathrm{C}$ with a 10 minute holding time to activate the polymerization reaction, followed by annealing at $400{ }^{\circ} \mathrm{C}\left(0.5^{\circ} \mathrm{C} / \mathrm{s}\right.$ with a 10 minute holding time $)$ in order to form the GNRs via cyclodehydrogenation.

\section{Scanning tunneling microscopy (STM)}

Topographic scanning tunneling microscopy images of as-grown AGNRs on Au $(11,12,12)$ and $\mathrm{Au}(788)$ were taken with a Scienta Omicron VT-STM operated at room temperature. Constant-current STM images were recorded with $1.5 \mathrm{~V}$ sample bias and $0.03 \mathrm{nA}$ setpoint current.

\section{GNR transfer and ex-situ characterization}

AGNRs were transferred from their growth gold substrate onto fused silica by electrochemical delamination method. First, PMMA was spin-coated (2500 rpm for 90 seconds) on GNR/Au to act as a support layer during the transfer. In a second step the PMMA/GNR/Au was heated at $80{ }^{\circ} \mathrm{C}$ for 10 minutes. In order to decrease the delamination time the PMMA on the edges of the Au substrate was etched by UVO exposure (80 minutes) followed by 3 minutes development in water: IPA.

The electrochemical delamination transfer was carried using $1 \mathrm{M} \mathrm{NaOH}$ aqueous solution as electrolyte. The electrochemical cell was mounted using a carbon rod as anode and the PMMA/GNRs/Au as the cathode. A DC current of $0.2 \mathrm{~A}(5 \mathrm{~V})$ was applied between anode and cathode during 1 minute. Hydrogen bubbles were formed in the cathode as a results of water reduction reaction. These bubbles permeates into the PMMA/GNRs and Au interface resulting in the detachment of the PMMA layer. The PMMA/GNR layer was cleaned for 5 minutes in ultrapure water before the transfer to the target substrate. After transfer onto fused silica PMMA/GNRs was baked for 10 minutes at $80^{\circ} \mathrm{C}$ followed by 20 minutes at $110^{\circ} \mathrm{C}$ in order to increase the adhesion between the target substrate and the PMMA/GNR layer. Finally, PMMA was dissolved by soaking it in acetone (15 minutes).

\section{Polarized Raman spectroscopy}


Polarized Raman spectra were performed with Witec Alpha 300 R Confocal Raman microscope, using 532 $\mathrm{nm}(2.33 \mathrm{eV})$ as excitation wavelength. The incident light was focused on the sample using a $50 \mathrm{x}$ long working distance objective with a numerical aperture NA $=0.55$, resulting in a spot size of about $600 \mathrm{~nm}$ FWHM. A motorized half wave plate was used to change the polarization direction of the incident laser beam from -90 to +90 degree, and a manual analyzing polarizer was kept parallel in the detection path. In order to improve signal to noise ratio Raman maps (10x10um) were obtained for GNRs on Au and on fused silica under vacuum $\left(1 \times 10^{-2}\right.$ mbar $) .^{40}$

\section{Atomic Force Microscopy}

The topography of transferred graphene nanoribbon samples was characterized using tapping mode AFM (Bioscope, Bruker) with silicon probes from OPUS (model 160AC-SG Ultrasharp Cantilever ) with tip radius $<1 \mathrm{~nm}$ (force constant $\sim 26 \mathrm{~N} \mathrm{~m}^{-1}$, resonance frequency in the range of $300 \mathrm{kHz}$ ). Height diagrams were recorded with scan sizes of $1.5 \mu \mathrm{m}$ and scan speeds of $1 \mathrm{~Hz}(512 \times 512$ points $)$. The WSxM software was used for AFM analysis. ${ }^{41}$

\section{Polarization spectroscopy technique}

Optical imaging and absorption spectroscopy measurements were carried out using a supercontinuum laser (YSL SC-PRO). Light polarization was controlled by a paired calcite polarizers (Glan-Thompson) and high polarization purity was achieved and retained in the imaging and spectroscopy (extinction ratio $>20,000$ ). Sample contrast was imaged by a standard Si CCD camera. Reflection contrast spectra $d R / R$ were obtained by measuring the reflectance at two close-by spots with and without GNRs with a piezoelectric motor. The light was collected into a grating spectrometer (Princeton Instruments) and InGaAs CCD (iDus $1.7 \mu \mathrm{m}$ InGaAs) with grating to obtain spectra in the visible and near-IR spectral range.

\section{Acknowledgments}

The authors would like to thank Juan Pablo Llinas, Dr. Kyunghoon Lee, Prof. Jeffrey Bokor, Prof. Felix R. Fischer and Prof. Michael F. Crommie in University of California at Berkeley for valuable discussions. The optical imaging and spectroscopy work and data analysis were supported by the Office of Naval research (MURI award N00014-16-1-2921) and by the NSF award 1808635. G.B.B, P.R and R.F acknowledge the support by the Swiss National Science Foundation under Grant No 20PC21_155644, the European Union's Horizon 2020 research and innovation programme under grant agreement number 785219 (Graphene Flagship Core 2), the Office of Naval Research (N00014-18-1-2708) and the NCCR MARVEL funded by the Swiss National Science Foundation (51NF40-182892).

Supporting Information is available on the ACS Publication website.

\section{Author contributions}

F.W. and R.F. supervised this project. S.Z. proposed and performed the imaging and spectroscopy measurements. G.B.B., J.O., R.D., M.C. and P.R. prepared and characterized the AGNR samples prior to imaging and spectroscopy. T.L., S.D. and S.W. helped in the optical measurements. T.C. and S.G.L. carried out the theoretical calculations. T.D., A.N. and K.M. prepreaed the precursors for GNR growth. All authors discussed the results and wrote the paper.

Competing interests. The authors declare no competing financial interests. 


\section{References}

(1) Cai, J.; Ruffieux, P.; Jaafar, R.; Bieri, M.; Braun, T.; Blankenburg, S.; Muoth, M.; Seitsonen, A. P.; Saleh, M.; Feng, X.; Mullen, K.; Fasel, R. Atomically precise bottom-up fabrication of graphene nanoribbons. Nature 2010, 466, 470-473.

(2) Ruffieux, P.; Cai, J.; Plumb, N. C.; Patthey, L.; Prezzi, D.; Ferretti, A.; Molinari, E.; Feng, X.; Mullen, K.; Pignedoli, C. A.; Fasel, R. Electronic structure of atomically precise graphene nanoribbons. ACS Nano 2012, 6, 6930-6935.

(3) Huang, H.; Wei, D.; Sun, J.; Wong, S.-L.; Feng, Y.-P.; Castro Neto, A. H.; Wee, A. T. S. Spatially resolved electronic structures of atomically precise armchair graphene nanoribbons. Sci. Rep. 2012, 2,983

(4) Linden, S.; Zhong, D.; Timmer, A.; Aghdassi, N.; Franke, J. H.; Zhang, H.; Feng, X.; Mullen, K.; Fuchs, H.; Chi, L.; Zacharias, H. Electronic Structure of Spatially Aligned Graphene Nanoribbons on $\mathrm{Au}(788)$. Phys. Rev. Lett. 2012, 108, 216801.

(5) Chen, Y.-C.; de Oteyza, D. G.; Pedramrazi, Z.; Chen, C.; Fischer, F. R.; Crommie, M. F. Tuning the band gap of graphene nanoribbons synthesized from molecular precursors. ACS Nano 2013, 7, $6123-6128$.

(6) Cai, J.; Pignedoli, C. A.; Talirz, L.; Ruffieux, P.; Söde, H.; Liang, L.; Meunier, V.; Berger, R.; Li, R.; Feng, X.; Müllen, K; Fasel, R. Graphene nanoribbon heterojunctions. Nat. Nanotechnol. 2014, 9, 896.

(7) Chen, Y.-C.; Cao, T.; Chen, C.; Pedramrazi, Z.; Haberer, D.; de Oteyza, D.; Fischer, F.; Louie, S.; Crommie, M. Molecular bandgap engineering of bottom-up synthesized graphene nanoribbon heterojunctions. Nat. Nanotechnol. 2015, 10, 156.

(8) Cloke, R. R.; Marangoni, T.; Nguyen, G. D.; Joshi, T.; Rizzo, D. J.; Bronner, C.; Cao, T.; Louie, S. G.; Crommie, M. F.; Fischer, F. R. Site-Specific Substitutional Boron Doping of Semiconducting Armchair Graphene Nanoribbons. J. Am. Chem. Soc. 2015, 137, 8872-8875.

(9) Kawai, S.; Saito, S.; Osumi, S.; Yamaguchi, S.; Foster, A. S.; Spijker, P.; Meyer, E. Atomically controlled substitutional boron-doping of graphene nanoribbons. Nat. Commun. 2015, 6, 8098.

(10) Kimouche, A.; Ervasti, M. M.; Drost, R.; Halonen, S.; Harju, A.; Joensuu, P. M; Sainio, J.; Liljeroth, P. Ultra-narrow metallic armchair graphene nanoribbons. Nat. Commun. 2015, 6, 10177.

(11) Ruffieux, P.; Wang, S.; Yang, B.; Sanchez-Sanchez, C.; Liu, J.; Dienel, T.; Talirz, L.; Shinde, P.; Pignedoli, C. A.; Passerone, D.; Dumslaff, T.; Feng, X.; Mullen, K.; Fasel, R. Onsurface synthesis of graphene nanoribbons with zigzag edge topology. Nature 2016, 531, 489-492.

(12) Mehdi Pour, M.; Lashkov, A.; Radocea, A.; Liu, X.; Sun, T.; Lipatov, A.; Korlacki, R. A.; Shekhirev, M.; Aluru, N. R.; Lyding, J. W.; Sysoev, V.; Sinitskii, A. Laterally Extended Atomically Precise Graphene Nanoribbons with Improved Electrical Conductivity for Efficient Gas Sensing. Nat. Commun. 2017, 8, 820.

(13) Talirz, L.; Söde, H.; Dumslaff, T.; Wang, S.; Sanchez-Valencia, J. R.; Liu, J.; Shinde, P.; Pignedoli, C. A.; Liang, L.; Meunier, V.; Plumb, N. C.; Shi, M.; Feng, X.; Narita, A.; Müllen, K.; Fasel, R.; Ruffieux, P. On-Surface Synthesis and Characterization of 9-Atom Wide Armchair Graphene Nanoribbons. ACS Nano 2017, 112, 1380-1388.

(14) Cao, T.; Zhao, F.; Louie, S. G. Topological Phases in Graphene Nanoribbons: Junction States, Spin Centers, and Quantum Spin Chains. Phys. Rev. Lett. 2017, 119, 076401.

(15) Rizzo, D. J.; Veber, G.; Cao, T.; Bronner, C.; Chen, T.; Zhao, F.; Rodriguez, H.; Louie, S. G.; Crommie, M. F.; Fischer, F. R. Topological band engineering of graphene nanoribbons. Nature 2018, 560, 204-208.

(16) Oliver Gröning, Shiyong Wang, Xuelin Yao, Carlo A. Pignedoli, Gabriela Borin Barin, Colin Daniels, Andrew Cupo, Vincent Meunier, Xinliang Feng, Akimitsu Narita, Klaus Müllen, Pascal Ruffieux \& Roman Fasel. Engineering of robust topological quantum phases in graphene nanoribbons. Nature 2018, 560, 209-213. 

manipulation of graphene nanoribbons. Nature 2018, 557, 691-695.

(18) Bennett, P. B.; Pedramrazi, Z.; Madani, A.; Chen, Y.-C.; de Oteyza, G. D.; Chen, C.; Fischer, F. R.; Crommie, M. F.; Bokor, J. Bottom-up graphene nanoribbon field-effect transistors. Appl. Phys. Lett. 2013, 103, 253114.

(19) Linas, J. P.; Fairbrother, A.; Barin, G.B.; Shi, W.; Lee, K.; Wu, S.; Choi, B. Y.; Pedramrazi, R. Z.; Dumslaff, T.; Narita, A.; Feng, X.; Müllen, K.; Fischer, F. R.; Zettl, A.; Ruffieux, P.; Yablonovitch, E.; Crommie, M. F.; Fasel, R.; Bokor, J. Short-channel field-effect transistors with 9-atom and 13-atom wide graphene nanoribbons. Nat. Commun. 2017, 8, 633.

(20) Muñoz, E.; Lu, J.; Yakobson, B. I. Ballistic Thermal Conductance of Graphene Ribbons. Nano Lett. 2010, 10, 1652-1656.

(21) Fairbrother, A.; Sanchez-Valencia, J.-R.; Lauber, B.; Shorubalko, I.; Ruffieux, P.; Hintermann, P.; Fasel, S. High vacuum synthesis and ambient stability of bottom-up graphene nanoribbons. Nanoscale 2017,9, 2785-2792

(22) Barin, G. B.; Fairbrother, A.; Rotach, L.; Bayle, M.; Paillet, M.; Liang, L.; Meunier, V.; Hauert, R.; Dumslaff, T.; Narita, A.; Mullen, K.; Sahabudeen, H.; Berger, R.; Feng, X; Fasel, R.; Ruffieux, P. Surface-Synthesized Graphene Nanoribbons for Room Temperature Switching Devices: Substrate Transfer and ex Situ Characterization. ACS Appl. Nano Mater. 2019, 2, 2184-2192

(23) Nakada, K.; Fujita, M.; Dresselhaus, G.; Dresselhaus, M. S. Edge state in graphene ribbons: Nanometer size effect and edge shape dependence. Phys. Rev. B 1996, 54, 17954-17961.

(24) Son, Y.-W.; Cohen, M. L.; Louie, S. G. Energy Gaps in Graphene Nanoribbons. Phys. Rev. Lett. 2006, 97, 216803.

(25) Barone, V.; Hod, O.; Scuseria, G. E. Electronic structure and stability of semiconducting graphene nanoribbons. Nano Lett. 2006, 6, 2748-2754.

(26) (6) Yang, L.; Park, C.-H.; Son, Y.-W.; Cohen, M. L.; Louie, S. G. Quasiparticle Energies and Band Gaps in Graphene Nanoribbons. Phys. Rev. Lett. 2007, 99, 186801.

(27) Verzhbitskiy, I. A.; De Corato, M.; Ruini, A.; Molinari, E.; Narita, A.; Hu, Y.; Schwab, M. G.; Bruna, M.; Yoon, D.; Milana, S.; Feng, X.; Müllen, K.; Ferrari, A. C.; Casiraghi, C.; Prezzi, D. Raman Fingerprints of Atomically Precise Graphene Nanoribbons. Nano Lett. 2016, $166,3442-3447$.

(28) Senkovskiy, B. V.; Pfeiffer, M.; Alavi, S. K.; Bliesener, A.; Zhu, J.; Michel, S.; Fedorov, A. V.; German, R.; Hertel, D.; Haberer, D.; Petaccia, L.; Fischer, F. R.; Meerholz, K.; van Loosdrecht, P. H. M.; Lindfors, K; Grüneis, A. Making Graphene Nanoribbons Photoluminescent. Nano Lett. 2017, 17, 4029-4037.

(29) Zhao, S.; Barin, G. B.; Rondin, L.; Raynaud, C.; Fairbrother, A.; Dumslaff, T.; Campidelli, S.; Müllen, K.; Narita, A.; Voisin, C.; Ruffieux, P.; Fasel, R.; Lauret, J.-S. Optical Investigation of On-Surface Synthesized Armchair Graphene Nanoribbons. Phys. Status Solidi B 2017, 254, 1700223.

(30) Denk, R.; Hohage, M.; Zeppenfeld, P.; Cai, J.; Pignedoli, C. A.; Söde, H.; Fasel, R.; Feng, X.; Müllen, K.; Wang, S.; Prezzi, D.; Ferretti, A.; Ruini, A.; Molinari, E.; Ruffieux, P. Exciton-dominated optical response of ultra-narrow graphene nanoribbons. Nat. Commun. 2014, 5,4253 .

(31) Denk, R.; Lodi-Rizzini, A.; Wang, S.; Hohage, M.; Zeppenfeld, P.; Cai, J.; Fasel, R.; Ruffieux, P.; Berger, R.; Chen, Z.; Narita, A.; Feng, X.; Müllen, K.; Biagi, R.; De Renzi, V.; Prezzi, D.; Ruini, A.; Ferrettic, A. Probing optical excitations in chevron-like armchair graphene nanoribbons. Nanoscale 2017, 9 (46). (1)

(32) Chen, Z.; Wang, H. I.; Teyssandier, J.; Dumsla, T.; Ivanov, I.; Zhang, W.; Ru, P.; Fasel, R.; Joachim, H.; Turchinovich, D.; De Feyter, S.; Feng, X.; Kläui, M.; Narita, A.; Bonn, M.; 
Klaus Müllen, K. Chemical Vapor Deposition Synthesis and Terahertz. J. Am. Chem. Soc. 2017, 139, 3635-3638.

(33) Li, G.; Yoon, K.-Y.; Zhong, X.; Zhu, X.; Dong, G. Efficient Bottom-Up Preparation of Graphene Nanoribbons by Mild Suzuki-Miyaura Polymerization of Simple Triaryl Monomers. Chem. Eur. J. 2016 22, 9116-9120.

(34) Cançado, L. G.; Pimenta, M. A.; Neves, B. R. A.; Medeiros-Ribeiro, G.; Enoki, T.; Kobayashi, Y.; Takai, K.; Fukui, K. I.; Dresselhaus, M. S.; Saito, R.; Jorio, A. Anisotropy of the Raman Spectra of Nanographite Ribbons. Phys. Rev. Lett. 2004, 93, 047403.

(35) Jorio, A.; Souza Filho, A. G.; Brar, V. W.; Swan, A. K.; Ünlü, M. S.; Goldberg, B. B.; Righi, A.; Hafner, J. H.; Lieber, C. M.; Saito, R.; Dresselhaus, G.; Dresselhaus, M. S. Polarized Resonant Raman Study of Isolated Single-Wall Carbon Nanotubes: Symmetry Selection Rules, Dipolar and Multipolar Antenna Effects. Phys. Rev. B 2002, 65, 121402(R).

(36) Liu, K.; Hong, X.; Zhou, Q.; Jin, C.; Li, J.; Zhou, W.; Liu, J.; Wang, E.; Zettl, A.; Wang, F. High-throughput optical imaging and spectroscopy of individual carbon nanotubes in devices. Nature Nanotechnol. 2013, 8, 917.

(37) Yang, Li; Cohen, M. L.; Louie, S. G. Excitonic Effects in the Optical Spectra of Graphene Nanoribbons. Nano Lett. 2007, 7, 3112-3115.

(38) Prezzi, D.; Varsano, D.; Ruini, A.; Marini, A.; Molinari, E. Optical properties of graphene nanoribbons: The role of many-body effects. Phys. Rev. B 2008 77, 041404.

(39) Di Giovannantonio, M.; Deniz, O.; Urgel, J. I.; Widmer, R.; Dienel, T.; Stolz, S.; Sanchez-Sa nchez, C.; Muntwiler, M.; Dumslaff, T.; Berger, R.; Narita, A.; Feng, X.; Müllen, K.; Ruffieux, P.; Fasel, R. On-Surface Growth Dynamics of Graphene Nanoribbons: The Role of Halogen Functionalization. ACS Nano 2018, 12, 74-81.

(40) Overbeck, J.; Barin, G. B.; Daniels, C.; Perrin, M.; Liang, L.; Braun, O.; Darawish, R.; Burkhardt, B.; Dumslaff, T.; Wang, X.-Y.; Narita, A.; Müllen, K.; Meunier, V.; Fasel, R.; Calame, M.; Ruffieux, P. Optimized substrates and measurement approaches for Raman spectroscopy of graphene nanoribbons. arxiv.org/abs/1907.01797.

(41) Horcas, I.; Fernandez, R.; Gomez-Rodríguez, J. M.; Colchero, J.; Gomez-Herrero, J.; Baro, A. M. WSXM: A software for scanning probe microscopy and a tool for nanotechnology. Rev. Sci. Instrum. 2007, 78, 013705. 